\title{
Associations Between Dynamic Contrast Enhanced Magnetic Resonance Imaging and Clinically Relevant Histopathological Features in Breast Cancer: A Multicenter Analysis
}

\author{
ALEXEY SUROV ${ }^{1}$, JIN YOU KIM ${ }^{2}$, MARCO AIELLO ${ }^{3}$, WEI HUANG ${ }^{4}$, \\ THOMAS E. YANKEELOV ${ }^{5}$, ANDREAS WIENKE $^{6}$ and MACIEJ PECH ${ }^{1}$ \\ ${ }^{1}$ Department of Radiology and Nuclear Medicine, Otto-von-Guericke-University Magdeburg, Magdeburg, Germany; \\ ${ }^{2}$ Medical Research Institute and Department of Radiology, \\ Pusan National University School of Medicine, Busan, Republic of Korea; \\ ${ }^{3}$ RCCS SDN, Istituto di Ricerca, Naples, Italy; \\ ${ }^{4}$ Advanced Imaging Research Center, Oregon Health \& Science University, Portland, OR, U.S.A.; \\ ${ }^{5}$ Institute of Imaging Science, Vanderbilt University, Nashville, TN, U.S.A.; \\ ${ }^{6}$ Institute of Medical Epidemiology, Biostatistics, and Informatics, \\ Martin-Luther-University Halle-Wittenberg, Halle, Germany
}

\begin{abstract}
Background/Aim: To provide data regarding relationships between quantitative dynamic contrast enhanced magnetic resonance imaging (DCE MRI) and prognostic factors in breast cancer (BC). Patients and Methods: Data from 4 Centers (200 female patients, mean age, $51.2 \pm 11.5$ years) were acquired. The following data were collected: histopathological diagnosis, tumor grade, stage, hormone receptor status, KI 67, and DCE MRI values including $K_{\text {trans }}$ (volume transfer constant), $V_{e}$ (volume of the extravascular extracellular leakage space (EES) and $K_{e p}$ (diffusion of contrast medium from the EES back to the plasma). DCE MRI values between different groups were compared using the Mann-Whitney U-test and by the Kruskal-Wallis $H$ test. The association between DCE MRI and Ki 67 values was calculated by the Spearman's rank correlation coefficient. Results: DCE MRI values of different tumor subtypes overlapped significantly. There were no statistically significant differences of DCE MRI values between different tumor grades. All DCE MRI parameters correlated with KI-67: $K_{\text {trans }}, r=0.44, p=0.0001 ; V_{e}, r=0.34$, $p=0.0001 ; K_{e p}, r=0.28, p=0.002$. ROC analysis identified $a$
\end{abstract}

This article is freely accessible online.

Correspondence to: Alexey Surov, Professor, MD, Department of Radiology and Nuclear Medicine, Otto-von-Guericke-University of Magdeburg, Leipziger Str. 44, 39112 Magdeburg, Germany. E-mail: Alexey.Surov@med.ovgu.de

Key Words: Breast cancer, DCE MRI, KI 67, hormone receptor.
$K_{\text {trans }}$ threshold of $0.3 \mathrm{~min}^{-1}$ for discrimination of tumors with low KI-67 expression (<25\%) and high KI-67 expression $(\geq 25 \%)$ : sensitivity, $75.5 \%$, specificity, $73.0 \%$, accuracy, $74.0 \%$, AUC, 0.78. DCE MRI values overlapped between tumors with different $T$ and $N$ stages. Conclusion: $K_{\text {trans }}, K_{e p}$, and $V_{e}$ cannot be used as reliable a surrogate marker for hormone receptor status, tumor stage and grade in BC. $K_{\text {trans }}$ may discriminate lesions with high and lower proliferation activity.

Breast cancer (BC) is the most common non cutaneous malignancy among women, representing 4 in 10 female cancer patients in the United States (1). Radiological imaging plays an essential role in the diagnosis and staging of BC. Moreover, imaging can also predict some clinically important histopathological features like expression of proliferation marker KI-67 (2, 3). So far, it has been shown that rim enhancement on dynamic magnetic resonance imaging (MRI) was associated with high expression of KI67 and poor prognosis of BC (3). Similarly, numerous studies analyzed the role of diffusion weighted imaging (DWI) in characterization of BC (4-7). Some authors observed statistically significant correlations between apparent diffusion coefficient and expression of KI-67 $(6,7)$, as well as with hormone receptor status (8). However, multicenter studies showed that ADC cannot reflect KI-67 and hormone receptor expression in $\mathrm{BC}(9,10)$.

Previously, some reports also indicated that dynamic contrast enhanced MRI (DCE MRI) can be used as imaging biomarker in $\mathrm{BC}(6,11,12,13)$. According to the literature, quantitative parameters of DCE MRI, namely volume transfer 
in vivo $36: 398-408(2022)$

Table I. Data regarding patient acquisition and technical details of breast DCE MRI in the involved Centers.

\begin{tabular}{|c|c|c|c|c|c|c|c|c|}
\hline Center & $\begin{array}{c}\text { Data } \\
\text { acquisition }\end{array}$ & $\begin{array}{c}\text { Patients, } \\
\text { n (\%) }\end{array}$ & $\begin{array}{c}\text { MR } \\
\text { scanner }\end{array}$ & $\begin{array}{c}\text { DCE } \\
\text { sequence }\end{array}$ & $\begin{array}{l}\mathrm{TR} / \mathrm{TE}, \\
\mathrm{ms}\end{array}$ & $\begin{array}{c}\text { Slice } \\
\text { thickness, mm }\end{array}$ & $\begin{array}{c}\text { Field of } \\
\text { view, mm }\end{array}$ & $\begin{array}{l}\text { Contrast } \\
\text { medium }\end{array}$ \\
\hline 1 & Prospective & $80(40.0 \%)$ & $\begin{array}{l}\text { 3T system (Trio Tim, } \\
\text { Siemens Healthcare, } \\
\text { Erlangen, Germany) }\end{array}$ & VIBE & $3.5 / 3.1$ & 2 & $320 \times 320$ & $\begin{array}{c}\text { gadobutrol (Gadovist; } \\
\text { Bayer Schering Pharma, } \\
\text { Berlin, Germany) }\end{array}$ \\
\hline 2 & Prospective & $42(21.0 \%)$ & $\begin{array}{l}\text { 3T system } \\
\text { (Biograph, Siemens } \\
\text { Healthcare, Erlangen, } \\
\text { Germany) }\end{array}$ & VIBE & $5.3 / 1.9$ & 3.6 & $356 \times 379$ & $\begin{array}{l}\text { Gadopentate dimeglumine } \\
\text { (Gd-DTPA; Magnevist, } \\
\text { Bayer Pharma AG, } \\
\text { Berlin, Germany }\end{array}$ \\
\hline 3 & Prospective & $49(24.5 \%)$ & $\begin{array}{l}\text { 3T system (Trio Tim, } \\
\text { Siemens Healthcare, } \\
\text { Erlangen, Germany) }\end{array}$ & TWIST & $6.2 / 2.9$ & 1.4 & $320 \times 320$ & $\begin{array}{c}\text { ProHance (Bracco } \\
\text { Diagnostic Inc.) }\end{array}$ \\
\hline 4 & Retrospective & $29(14.5 \%)$ & $\begin{array}{l}\text { Philips 3T Achieva } \\
\text { MR scanner } \\
\text { (Philips Healthcare, } \\
\text { Best, The Netherlands) }\end{array}$ & $\begin{array}{l}\text { RF-spoiled } \\
\text { 3D gradient } \\
\quad \text { echo }\end{array}$ & $7.9 / 1.3$ & 5 & $220 \times 220$ & $\begin{array}{c}\text { Gadopentate dimeglumine } \\
\text { (Gd-DTPA; Magnevist } \\
\text { Wayne, NJ) }\end{array}$ \\
\hline
\end{tabular}

DCE MRI, Dynamic contrast enhanced magnetic resonance imaging; VIBE, volumetric interpolated breath-hold examination; TWIST, time-resolved angiography with stochastic trajectories; RF, radio frequency.

constant $\left(\mathrm{K}_{\text {trans }}\right)$, volume of the extravascular extracellular leakage space $\left(\mathrm{V}_{\mathrm{e}}\right)$, and diffusion of contrast medium from the EES back to the plasma $\left(\mathrm{K}_{\mathrm{ep}}\right)$ reflect different histopathological features in $\mathrm{BC}(6,12,13)$. For example, Kang et al. showed that triple-negative $\mathrm{BC}$ exhibited higher $\mathrm{K}_{\text {trans }}$ and $\mathrm{K}_{\mathrm{ep}}$ in comparison to luminal cancers $(p<0.05)$ (12). Furthermore, estrogen receptor (ER) negative tumors had higher $\mathrm{K}_{\text {trans }}$ than ER-positive tumors $(p<0.05)$ and progesterone receptor (PR)-negative tumors presented higher $\mathrm{V}_{\mathrm{e}}$ than PR-positive tumors $(p<0.05)(12)$. Finally, tumors with higher KI-67 showed higher $\mathrm{K}_{\mathrm{ep}}$ than tumors with lower Ki-67 $(p<0.05)$ (12). Nagasaka et al. reported that the mean of $\mathrm{V}_{\mathrm{e}}$ was lower in cancers with a high KI-67 index than in cancers with low KI-67 ( $p=0.002)(13)$. However, other authors did not find any significant association between $\mathrm{V}_{\mathrm{e}}$, $\mathrm{K}_{\mathrm{ep}}$ and expression of KI-67 in BC (14).

The purpose of the present study was to provide evident data on relationships between DCE MRI parameters and clinically relevant histopathological features in BC.

\section{Patients and Methods}

Data acquisition and patients. The present analysis was approved by the institutional review board (Number: 36/20, Otto-vonGuericke University, Magdeburg).

For analysis of associations between imaging and histopathology in BC a multicenter work group was established $(9,10)$. For this study, the partners of our work group were contacted via email with the request to provide the data regarding DCE MRI in BC. There were the following Centers:

- Medical Research Institute, Pusan National University School of Medicine, Busan, Republic of Korea and Department of Radiology, Pusan National University Hospital, Busan, Republic of Korea (center 1);
Table II. Tumor subtypes.

\begin{tabular}{lc}
\hline Subtype & $\mathrm{n}(\%)$ \\
\hline Invasive ductal carcinoma & $167(83.5)$ \\
Invasive lobular carcinoma & $6(3.0)$ \\
Mucinous breast cancer & $3(1.5)$ \\
No special type & $24(12.0)$ \\
Receptor status & \\
Luminal A & $49(24.5)$ \\
Luminal B & $84(42.0)$ \\
HER 2+ & $32(16.0)$ \\
Triple negative & $19(9.5)$ \\
Not available & $16(8.0)$ \\
Tumor grade & \\
1 & $25(12.5)$ \\
2 & $96(48.0)$ \\
3 & $79(39.5)$ \\
T stage & \\
1 & $73(36.5)$ \\
2 & $78(39.0)$ \\
3 & $22(11.0)$ \\
4 & $27(13.5)$ \\
N stage & \\
0 & $104(52.0)$ \\
1 & $51(25.5)$ \\
2 & $22(11.0)$ \\
3 & $23(11.5)$ \\
M stage & $186(93.0)$ \\
0 & $14(7.0)$ \\
1 &
\end{tabular}

HER, Human epidermal growth factor receptor.

- RCCS SDN, Istituto di Ricerca, Naples, Italy (center 2);

- Advanced Imaging Research Center, Oregon Health \& Science University, Portland, OR, USA (center 3); 
Table III. DCE MRI values in BC with different hormone receptor status.

\begin{tabular}{lcccc}
\hline & Luminal A cancer & Luminal B cancer & HER 2+ cancer & Triple negative cancer \\
\hline $\mathrm{K}_{\text {trans }}, \min ^{-1}$ & $0.24 \pm 0.27$ & $0.44 \pm 0.44$ & $0.22 \pm 0.50$ & $0.53 \pm 1.71$ \\
$\mathrm{~V}_{\mathrm{e}}, \%$ & $0.61 \pm 0.43$ & $0.30 \pm 0.23$ & $0.44 \pm 0.44$ \\
$\mathrm{~K}_{\mathrm{ep}}, \mathrm{min}^{-1}$ & $0.50 \pm 0.43$ & $0.72 \pm 0.53$ & $0.56 \pm 0.58$ & $0.62 \pm 1.33$ \\
\hline
\end{tabular}

DCE MRI, Dynamic contrast enhanced magnetic resonance imaging; BC, breast cancer; HER, human epidermal growth factor receptor.

- Departments of Biomedical Engineering, Diagnostic Medicine, and Oncology, Livestrong Cancer Institutes, Oden Institute of Computational and Engineering Sciences, The University of Texas at Austin, USA (center 4).

For every case of the investigated patients/tumors the following data were collected: age, precise histopathological diagnosis, tumor grade, tumor stage, hormone receptor status, KI-67 index, and DCE MRI values including $\mathrm{K}_{\text {trans }}$ or volume transfer constant, $\mathrm{V}_{\mathrm{e}}$ or volume of the extravascular extracellular leakage space (EES) and $\mathrm{K}_{\mathrm{ep}}$ or diffusion of contrast medium from the EES back to the plasma.

The acquired sample comprises 200 patients (Table I). In every case, breast MRI was performed on a clinical scanner with dedicated breast radiofrequency coil. MR scanners and imaging protocols varied across the centers.

Statistical analysis. Continuous variables were described by mean value and standard deviation. Categorical variables were given as relative frequencies. The comparison of DCE MRI values in groups was performed by Mann-Whitney $U$-tests where the $p$-values are adjusted for multiple testing (Bonferroni correction). The association between DCE MRI values and KI-67 values was calculated by Spearman's rank correlation coefficient. Sensitivity, specificity, negative and positive predictive values, accuracy, and area under the receiver operating characteristic curve (AUC) value were calculated for the diagnostic procedures. Thresholds are chosen to maximize the Youden index

\section{Results}

Patients and tumors. A total of 200 female patients, mean age, 51.2 \pm 11.5 years were included in this study. The patients had a variety of different breast tumor histologic types (Table II). The majority of tumors were invasive ductal carcinoma (IDC, $81.95 \%$ ) with a limited number of other histopathological subypes. The DCE MRI values $(\mathrm{M} \pm \mathrm{SD})$ of the tumors were as follows: $\mathrm{K}_{\text {trans }}, 0.33 \pm 0.65 \mathrm{~min}^{-1} ; \mathrm{V}_{\mathrm{e}}$, $0.48 \pm 0.41 \% ; \mathrm{K}_{\mathrm{ep}}, 0.60 \pm 0.60 \mathrm{~min}^{-1}$.

DCE MRI and hormone receptor status. Hormone receptor status was available for 184 cases. Most frequently, luminal $\mathrm{B}$ cancers were diagnosed (Table III). DCE MRI values in different BC subtypes are given in Table IV. Triple-negative cancers had highest $\mathrm{K}_{\text {trans }}$ values, luminal B cancers had highest $\mathrm{K}_{\mathrm{ep}}$ and $\mathrm{V}_{\mathrm{e}}$ values, and HER 2+ BC had lowest $\mathrm{K}_{\text {trans }}$ and $\mathrm{V}_{\mathrm{e}}$ values. There were no significant differences between $\mathrm{K}_{\text {trans }}$ and $\mathrm{K}_{\mathrm{ep}}$ values in the $\mathrm{BC}$ subtypes. $\mathrm{V}_{\mathrm{e}}$
Table IV. DCE MRI values in BC with different tumor grades.

\begin{tabular}{lcccc}
\hline & Grade 1 & Grade 2 & Grade 3 & $p$-Value \\
\hline $\mathrm{K}_{\text {trans }}$, min $^{-1}$ & $0.12 \pm 0.10$ & $0.32 \pm 0.44$ & $0.42 \pm 0.90$ & 0.135 \\
$\mathrm{~V}_{\mathrm{e}}, \%$ & $0.35 \pm 0.29$ & $0.49 \pm 0.39$ & $0.52 \pm 0.46$ & 0.225 \\
$\mathrm{~K}_{\mathrm{ep}}, \min ^{-1}$ & $0.34 \pm 0.16$ & $0.60 \pm 0.54$ & $0.67 \pm 0.75$ & 0.064 \\
\hline
\end{tabular}

DCE MRI, Dynamic contrast enhanced magnetic resonance imaging; $\mathrm{BC}$, breast cancer.

values were different among the tumors with several receptor expressions $(p=0.003)$. However, all DCE MRI values of different tumor subtypes overlapped significantly (Figure 1).

DCE MRI and tumor grade. DCE MRI values in different tumor grades are given in Table IV. All DCE MRI values increased with tumor grade. However, there were no significant differences of DCE MRI values between several tumor grades and all DCE MRI values of different tumor types overlapped significantly (Figure 2).

DCE MRI and KI-67. The level of proliferation index KI-67 was available for 123 tumors. The mean value was $27.4 \pm 23.6 \%$, median value $=20 \%$, range $=1 \%-90 \%$. All DCE MRI parameters correlated with KI-67. The correlation coefficients were as follows: $\mathrm{K}_{\text {trans }}, \mathrm{r}=0.44, p=0.0001 ; \mathrm{V}_{\mathrm{e}}$, $\mathrm{r}=0.34, p=0.0001 ; \mathrm{K}_{\mathrm{ep}}, \mathrm{r}=0.28, p=0.002$. On the next step, ROC analysis was performed for distinguishing tumors with high proliferative potential from tumors with low proliferation rate using DCE MRI values. A KI-67 value of $25 \%$ was used as the threshold for discrimination between tumors with low KI-67 expression $(<25 \%)$ and high KI 67 expression $(\geq 25 \%)$. The Youden index identified threshold values of $\mathrm{K}_{\text {trans }}, \mathrm{V}_{\mathrm{e}}$, and $\mathrm{K}_{\mathrm{ep}}$ (Table $\mathrm{V}$ ). $\mathrm{K}_{\text {trans }}$ cut-off value of $0.3 \mathrm{~min}^{-1}$ showed best results (Figure 3). Furthermore, other threshold values of KI 67 ranging from $10 \%$ to $50 \%$ were also analyzed (Table VI). DCE MRI values had low area under the curve for every KI-67 threshold.

DCE MRI and $T$ stage. DCE MRI values differed significantly among the tumors with different $\mathrm{T}$ stages 

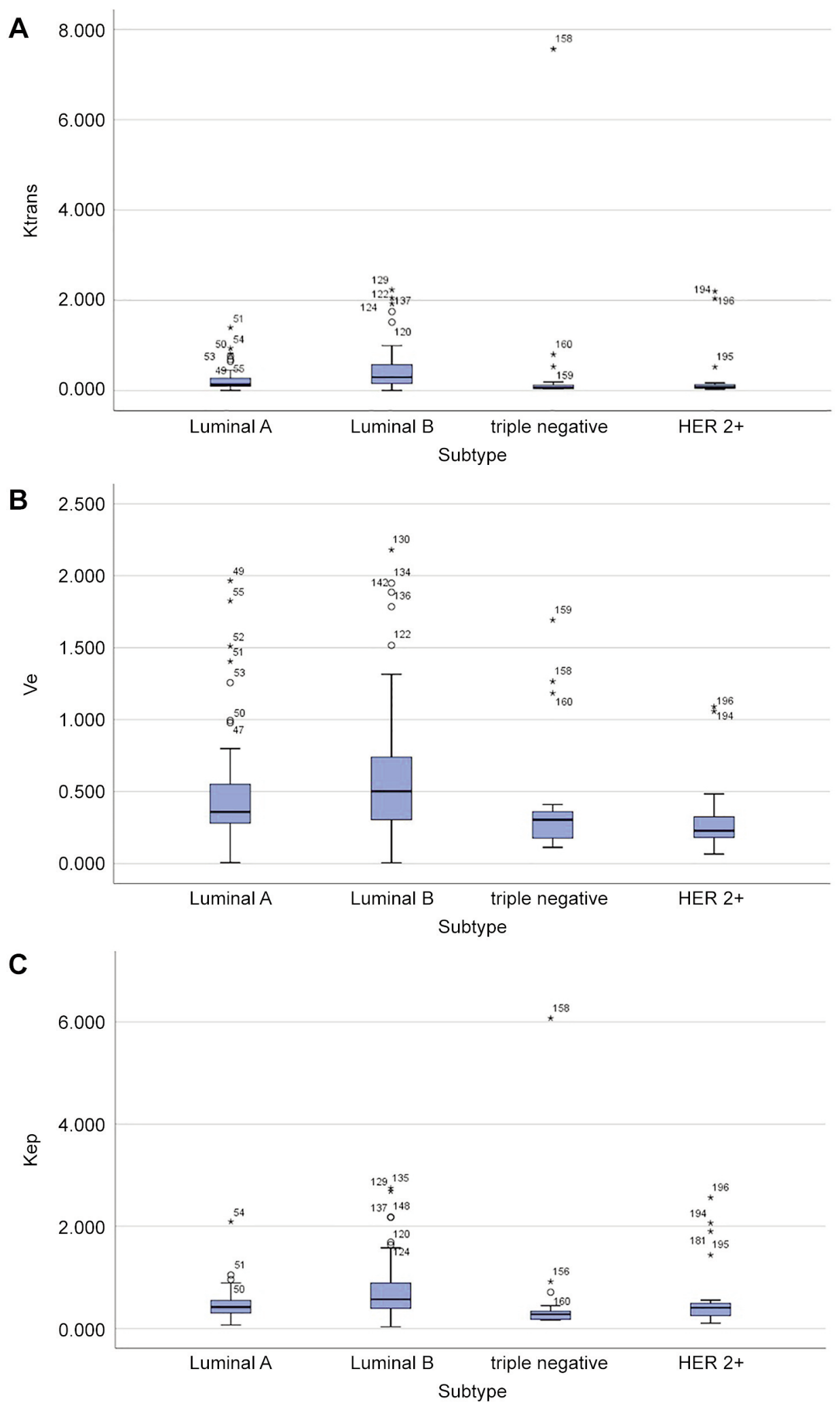

Figure 1. Box plots of DCE MRI values in tumors with different molecular subtypes. (A) $K_{\text {trans. }}$ No significant differences between $K_{\text {trans }}$ values in the $B C$ subtypes were found. (B) Overlapping of the $V_{e}$ values between the tumor subtypes. $(C) K_{e p}$. No significant differences between $K_{e p}$ values in the $B C$ subtypes were identified. 

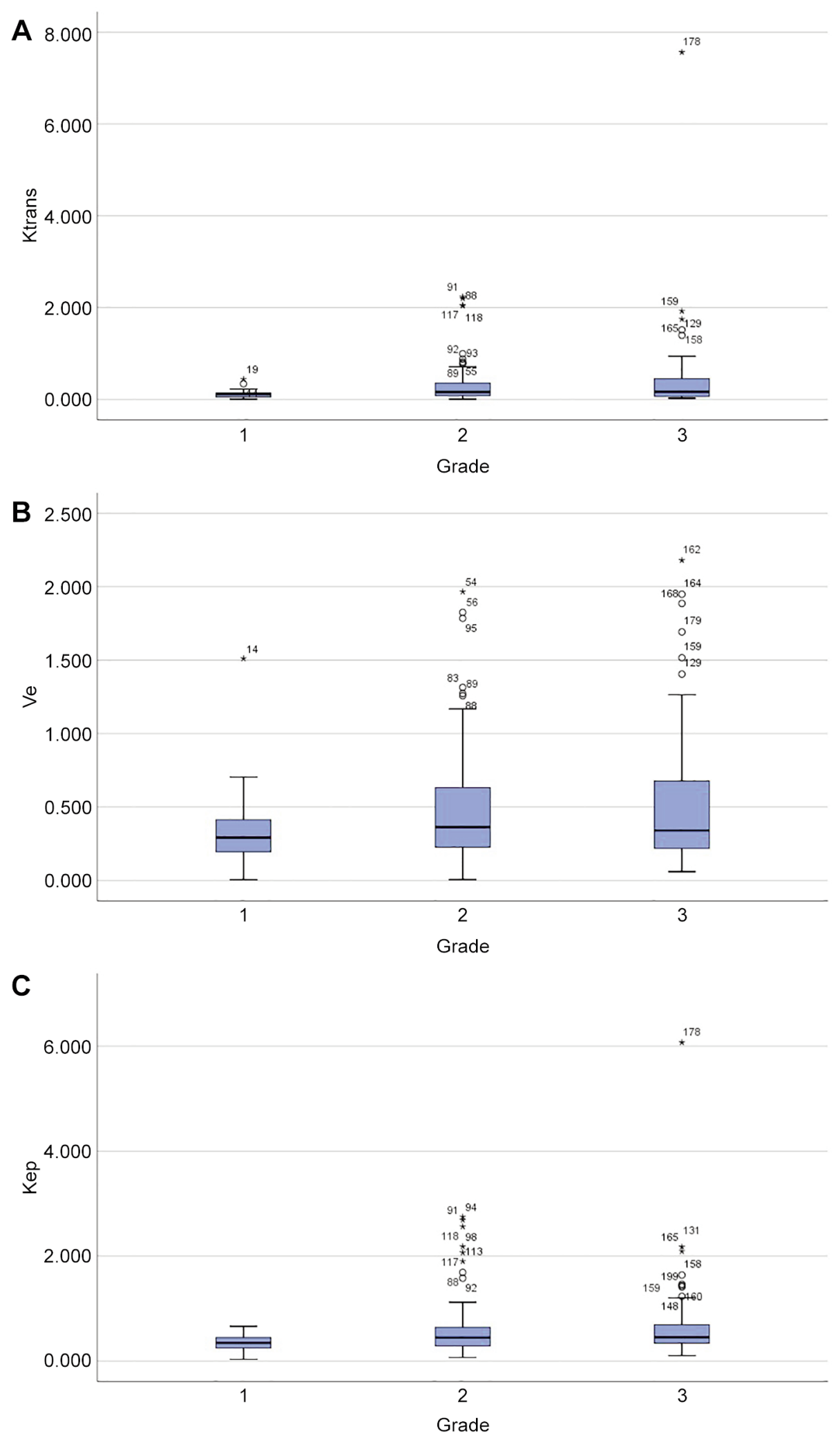

Figure 2. Box plots of DCE MRI values in carcinomas with different tumor grades. (A) $K_{\text {trans. }}$ There were no significant differences of $K_{\text {trans }}$ values between several tumor grades. (B) $V_{e}$. No significant differences of $V_{e}$ values between several tumor grades were observed. $(C) K_{e p} . K_{e p} v a l u e s$ did not differ significantly between several tumor grades. 
in vivo $36: 398-408(2022)$

Table V. Threshold values of Ktrans, Ve, and Kep for discrimination of tumors with high (>25\%) expression of KI-67.

\begin{tabular}{|c|c|c|c|c|c|c|}
\hline & Threshold & Sensitivity & Specificity & PPV & NPV & Accuracy \\
\hline $\mathrm{K}_{\text {trans }}, \min ^{-1}$ & 0.3 & $75.5 \%$ & $73.0 \%$ & $64.9 \%$ & $81.8 \%$ & $74.0 \%$ \\
\hline $\mathrm{v}_{\mathrm{e}}, \%$ & 0.5 & $73.5 \%$ & $66.2 \%$ & $59.0 \%$ & $79.0 \%$ & $69.1 \%$ \\
\hline $\mathrm{K}_{\mathrm{ep}}, \min ^{-1}$ & 0.55 & $63.3 \%$ & $63.5 \%$ & $53.4 \%$ & $72.3 \%$ & $63.4 \%$ \\
\hline
\end{tabular}

PPV, Positive predictive value; NPV, negative predictive value.

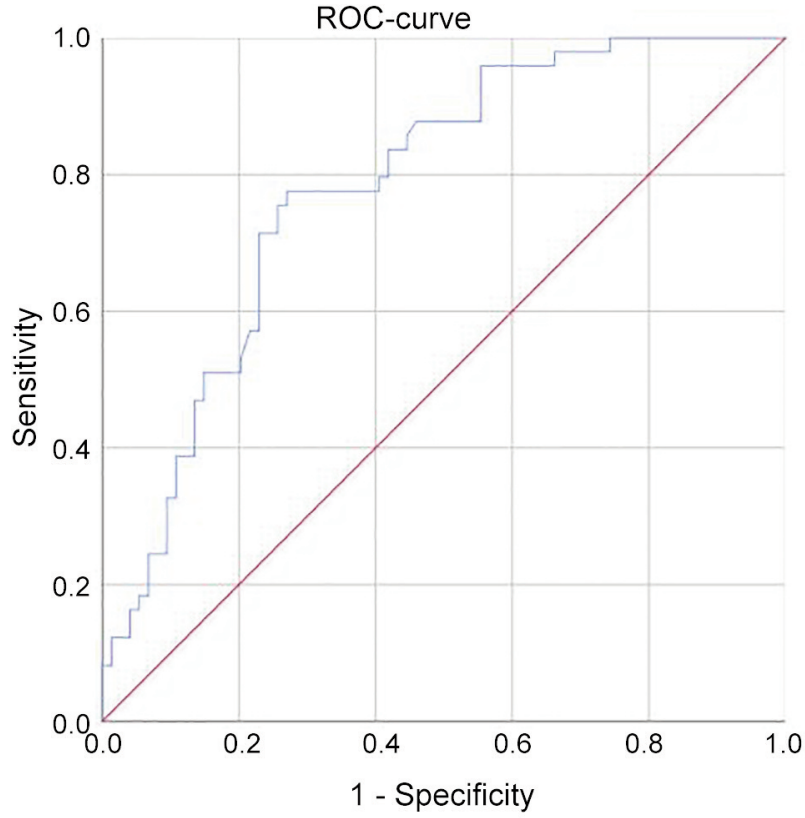

Figure 3. Receiver operating characteristic (ROC) curve for use of $K_{\text {trans }}$ for distinguishing of carcinomas with high proliferation potential (Ki67>25\%) from tumors with low Ki 67 level $(<25 \%)$. The area under the curve is 0.78 . The optimal threshold value is $0.3 \mathrm{~min}^{-}$ ${ }^{1}$ yealding a sensitivity of $75.5 \%$, a specificity of $73.0 \%$, an accuracy of $74.0 \%$. The positive predictive value is $64.9 \%$, and the negative predictive value is $81.8 \%$.

(Table VII). Carcinomas with T4 stage showed highest DCE MRI values in comparison to other tumor stages. However, DCE MRI values overlapped between the subgroups (Figure 4).

DCE MRI and nodal stage. Overall, in $104 \mathrm{BC}$ N0 and in $96 \mathrm{BC} \mathrm{N}+$ stages were diagnosed. All DCE MRI values were statistically significant higher in $\mathrm{N}+$ tumors than in N0 lesions (Table VIII). However, the graphical distribution of DCE MRI values showed that they overlapped between the subgroups (Figure 5). Furthermore, ROC analysis also showed that DCE MRI values had very low areas under the curve in prediction of nodal stage in BC (Figure 6).
Table VI. Areas under the curve for discrimination of tumors with different expression of KI-67 based on DCE MRI values.

\begin{tabular}{llllll}
\hline & \multicolumn{5}{c}{ KI-67 level } \\
\cline { 2 - 6 } & $\geq 10 \%$ & $\geq 20 \%$ & $\geq 30 \%$ & $\geq 40 \%$ & $\geq 50 \%$ \\
\hline $\mathrm{K}_{\text {trans }}$ & 0.72 & 0.77 & 0.70 & 0.74 & 0.68 \\
$\mathrm{~V}_{\mathrm{e}}$ & 0.67 & 0.73 & 0.64 & 0.63 & 0.55 \\
$\mathrm{~K}_{\mathrm{ep}}$ & 0.63 & 0.67 & 0.67 & 0.72 & 0.70 \\
\hline
\end{tabular}

DCE MRI, Dynamic contrast enhanced magnetic resonance imaging.

Table VII. DCE MRI values in breast cancer with different tumor $(T)$ stages.

\begin{tabular}{lccccc}
\hline & $\mathrm{T} 1$ & $\mathrm{~T} 2$ & $\mathrm{~T} 3$ & $\mathrm{~T} 4$ & $p$-Value \\
\hline $\mathrm{K}_{\text {trans }}, \mathrm{min}^{-1}$ & $0.18 \pm 0.13$ & $0.27 \pm 0.37$ & $0.14 \pm 0.17$ & $1.08 \pm 1.44$ & 0.001 \\
$\mathrm{~V}_{\mathrm{e}}, \%$ & $0.37 \pm 0.19$ & $0.44 \pm 0.39$ & $0.36 \pm 0.33$ & $0.99 \pm 0.57$ & 0.001 \\
$\mathrm{~K}_{\mathrm{ep}}, \mathrm{min}^{-1}$ & $0.50 \pm 0.29$ & $0.54 \pm 0.41$ & $0.36 \pm 0.23$ & $1.21 \pm 1.26$ & 0.001 \\
\hline
\end{tabular}

DCE MRI, Dynamic contrast enhanced magnetic resonance imaging.

Table VIII. Comparison of DCE MRI values in BC with and without nodal metastases.

\begin{tabular}{lccc}
\hline & $\begin{array}{c}\text { BC with N0 stage, } \\
\mathrm{M} \pm \mathrm{SD}\end{array}$ & $\begin{array}{c}\text { BC with N+ stage, } \\
\mathrm{M} \pm \mathrm{SD}\end{array}$ & $p$-Value \\
\hline $\mathrm{K}_{\text {trans }}, \mathrm{min}^{-1}$ & $0.17 \pm 0.15$ & $0.50 \pm 0.89$ & 0.001 \\
$\mathrm{~V}_{\mathrm{e}}, \%$ & $0.37 \pm 0.21$ & $0.60 \pm 0.52$ & 0.001 \\
$\mathrm{~K}_{\mathrm{ep}}, \mathrm{min}^{-1}$ & $0.48 \pm 0.34$ & $0.72 \pm 0.79$ & 0.007 \\
\hline
\end{tabular}

DCE MRI, Dynamic contrast enhanced magnetic resonance imaging; $\mathrm{BC}$, breast cancer.

\section{Discussion}

The present study is the first multicenter project regarding associations between DCE MRI and clinically relevant histopathological features in BC. Previously, the role of DCE MRI was analyzed systematically in prostate cancer, glioma, 

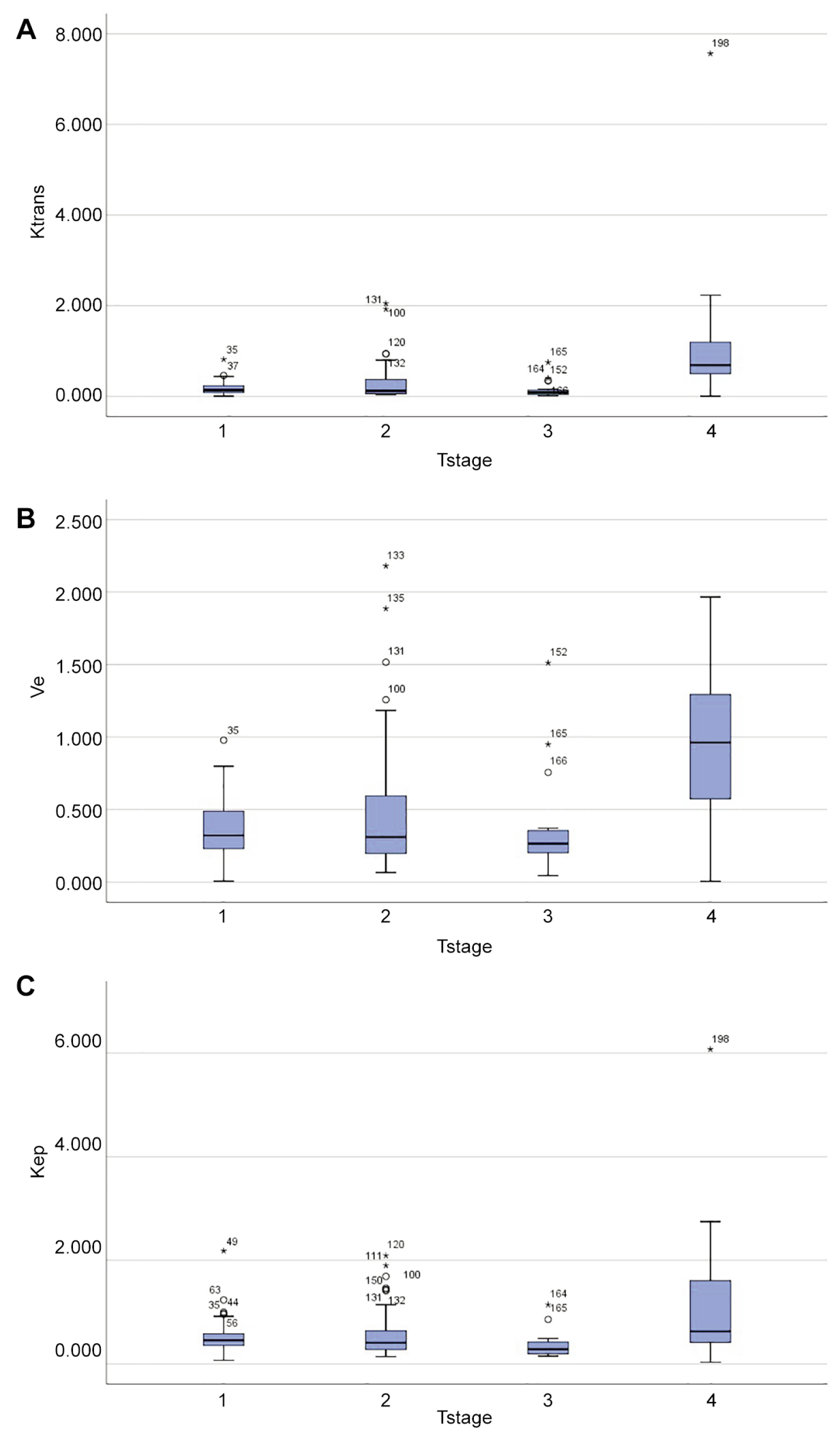

Figure 4. Box plots of DCE MRI values in carcinomas with different tumor stages. (A) $K_{\text {trans. }} K_{\text {trans }}$ values overlapped between the subgroups. (B) Significant overlapping of $V_{e}$ values between the different tumor stages. $(C)$ Overlapping of $K_{e p}$ values between the different tumor stages. 

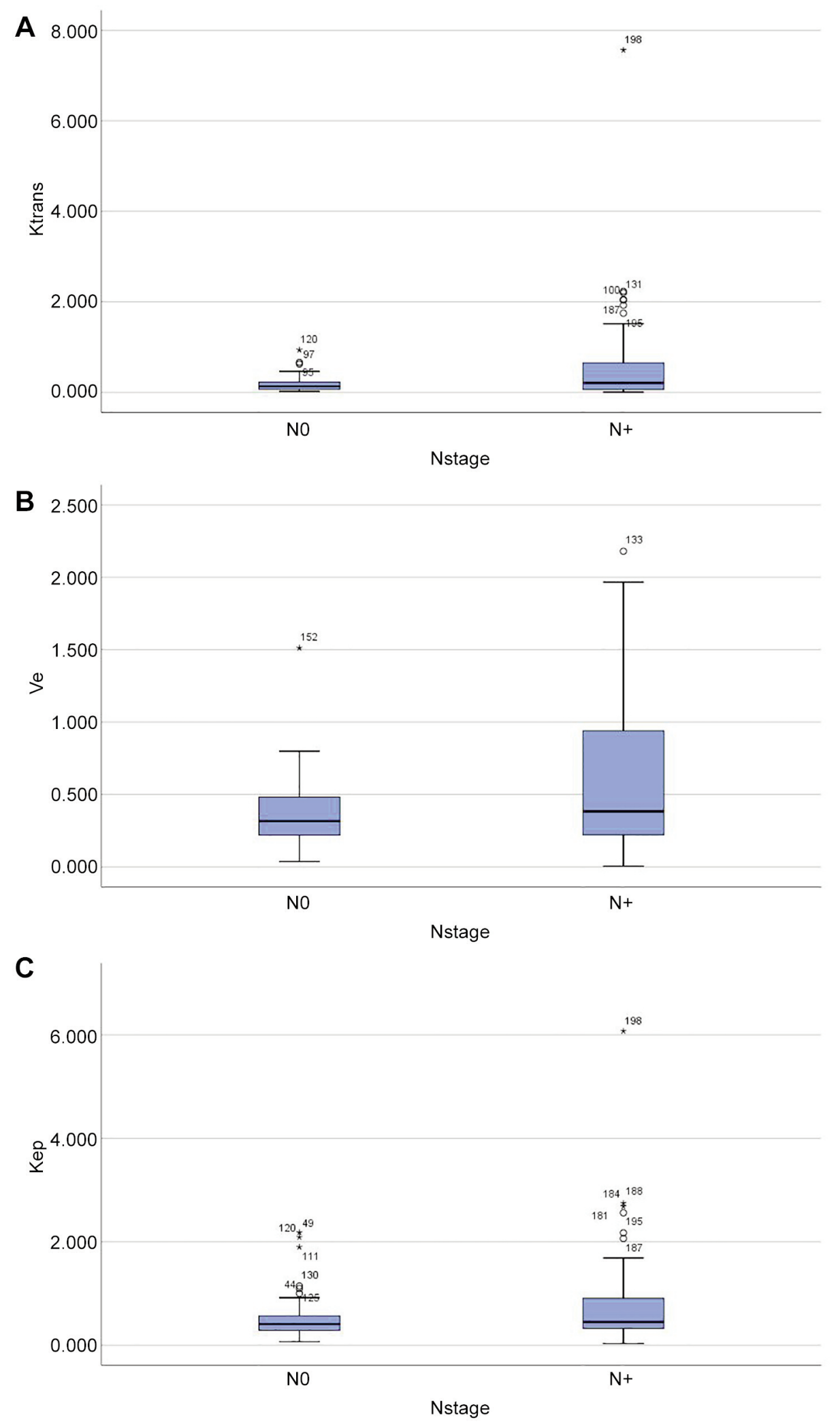

Figure 5. Box plots of DCE MRI values in carcinomas with different nodal stages (NO, tumors without nodal metastases; $N+$ tumors with nodal metastases). (A) Graphical distribution of $K_{\text {trans }}$ values showing overlapping between the subgroups. (B) Significant overlapping of $V_{e}$ values between the tumors with different nodal stages. (C) Significant overlapping of $K_{e p}$ values between the NO and $N+$ tumors. 


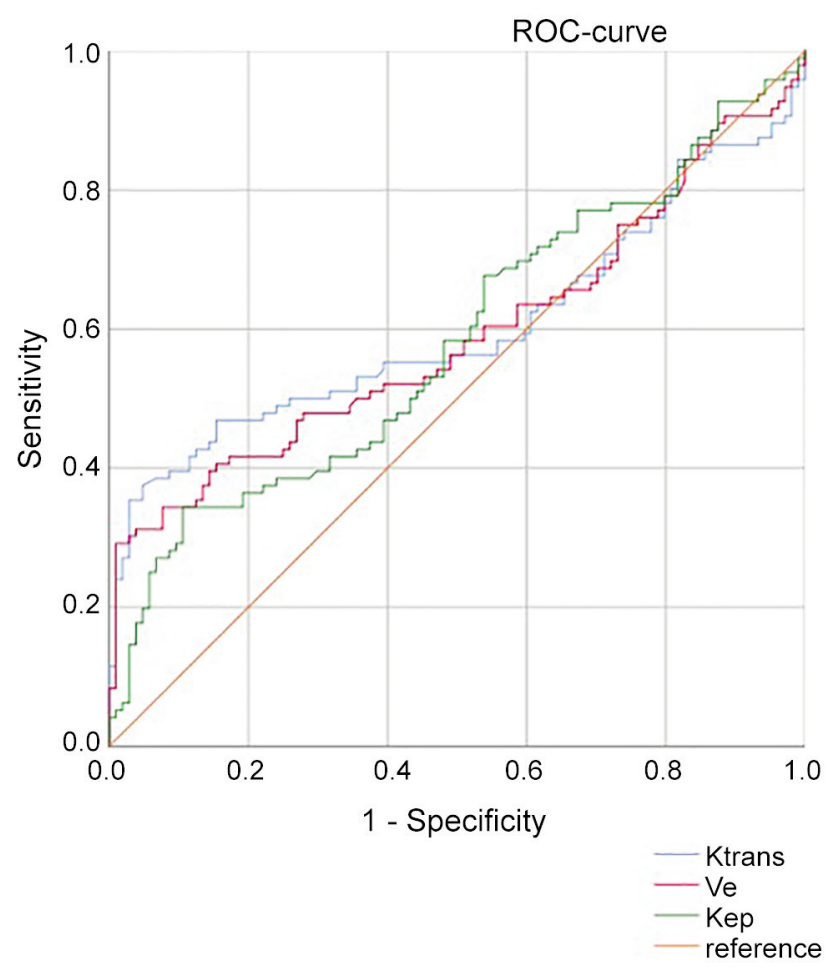

Figure 6. Receiver operating characteristic (ROC) curve for use of DCE $M R I$ values for distinguishing of carcinomas with and without nodal metastases. The areas under the curve for the parameters are as follows: $K_{\text {trans }}, 0.60 ; V_{e}, 0.59 ; K_{e p}, 0.58$.

and squamous cell carcinoma of the head and neck region. In prostate cancer, it was shown that $\mathrm{K}_{\text {trans }}$ values were significantly higher for high-grade versus low-grade lesions (15). Furthermore, $\mathrm{K}_{\mathrm{ep}}$ correlated positively with mean blood vessel count and mean vessel area (16). In glioma, $\mathrm{K}_{\text {trans }}$ and $\mathrm{V}_{\mathrm{e}}$ values of grade 2 tumors were significantly lower than those of grade 3 (17). Moreover, $\mathrm{K}_{\text {trans }}$ and $\mathrm{V}_{\mathrm{e}}$ significantly correlated with the KI-67 index (17). Finally, $\mathrm{K}_{\text {trans }}$ showed a significant positive correlation with microvessel density in different tumors (18). Also, in head and neck squamous cell carcinomas DCE MRI can predict relevant histopathological features. So far, $\mathrm{K}_{\text {trans }}$ correlated well with expression of KI67 and $\mathrm{V}_{\mathrm{e}}$ with the mean microvessel diameter (19).

In $\mathrm{BC}$, only few studies reported data about associations between DCE MRI and histopathology. The published results are promising. For instance, it has been shown that parameters of DCE MRI were different in tumors with different hormone receptor expression and grade. So far, grade 3 cancers had higher $K_{\text {trans }}$ and $K_{e p}$ values in comparison to grade 1 lesions $(11,12)$. Mean $\mathrm{V}_{\mathrm{e}}$ was lower in tumors with a high histologic grade than in tumors with a low histologic grade (11). Regarding expression of hormone receptors, triple negative $\mathrm{BC}$ showed higher $\mathrm{K}_{\text {trans }}$ and $\mathrm{K}_{\mathrm{ep}}$, but lower $\mathrm{V}_{\mathrm{e}}$ values than luminal $\mathrm{BC}$ (12). Furthermore, $\mathrm{V}_{\mathrm{e}}$ correlated inversely with HER 2 expression (20).

Our data showed that HER 2 rich BC had lowest $\mathrm{K}_{\text {trans }}$ and $\mathrm{V}_{\mathrm{e}}$ values in comparison to other subtypes. Furthermore, triple-negative $\mathrm{BC}$ had highest $\mathrm{K}_{\text {trans }}$ values. However, as shown, values of DCE MRI overlapped significantly and, therefore, cannot be used for prediction of hormone receptor status in BC in clinical practice. Furthermore, our data indicated that DCE MRI parameters did not reflect tumor grade in $\mathrm{BC}$.

Another important aspect in BC is expression of proliferation marker KI-67. It is well known that high expression of KI-67 is associated with a greater risk of death compared with lower expression rates (21). Therefore, prediction of proliferation potential of $\mathrm{BC}$ based on imaging is very important. According to previous reports, parameters of DCE MRI are associated with the KI-67 index. However, the reported data are controversial $(12,13,21)$. For example, Kang et al. showed that carcinomas with high expression KI67 showed statistically significant higher Kep values in comparison to $\mathrm{BC}$ with low expression of $\mathrm{KI}-67$ and $\mathrm{K}_{\text {trans }}$ and $\mathrm{V}_{\mathrm{e}}$ values did not differ between the tumors (12). Liu et al. found that only $\mathrm{K}_{\text {trans }}$ correlated with KI-67 (20). Koo et al. did not observe any statistically significant associations between DCE MRI values and KI-67 (11). Finally, Kim et al. identified significant relationships between $\mathrm{K}_{\text {trans }}, \mathrm{K}_{\mathrm{ep}}$ and KI-67, but not between $\mathrm{V}_{\mathrm{e}}$ and KI-67 (22). Moreover, the previous studies used different thresholds of KI 67 expression for distinguishing tumors with low and high proliferation activity, namely $15 \%(12), 10 \%$ (20), and $5 \%$ (11). In one study two threshold values, $5 \%$ and $15 \%$ were analyzed (22). This fact relativizes the reported results. According to a large meta-analysis based on data of 64,196 patients, the optimal KI-67 cut-off is $25 \%$ (21). It has been shown that this cut-off is associated with a greater risk of death compared with lower expression rates (21).

In the present work, KI-67 correlated well with all DCE MRI parameters. The strongest correlation was observed with $\mathrm{K}_{\text {trans }}(\mathrm{r}=0.44, p=0.0001)$. However, the optimal threshold of $\mathrm{K}_{\text {trans }}$ to discriminate $\mathrm{BC}$ with high (>25\%) and low $(<25 \%)$ expression of KI-67 yielded a relatively low sensitivity $(75.5 \%)$ and specificity $(73.0 \%)$, as well as low accuracy $(74.0 \%)$. Also, this applied for several alternate thresholds of KI-67 expression ranging from $10 \%$ to $50 \%$.

Another important clinical question is, if imaging features of primary tumors can predict occurrence of nodal and/or distant metastases. Previously, it was indicated that some MRI features of BC were associated with occurrence of nodal metastases (23). Regarding DCE MRI, presumably, perfusion parameters of primary tumor may be able to predict occurrence of lymph node metastases. In fact, BC with lymphovascular space invasion (LVSI) had higher $\mathrm{K}_{\text {trans }}$ and $\mathrm{K}_{\mathrm{ep}}$ than tumors without LVSI (12). Our results, 
however, did not confirm this hypothesis. Although DCE MRI parameters of BC differed between N0 vs. $\mathrm{N}+$ stages, all of them overlapped significantly. Therefore, parameters of DCE MRI obtained from primary tumors cannot be used for prediction of nodal stage in BC.

The present multicenter study is the largest to date. However, there are certain limitations to address. The involved patients were investigated on different MR scanners with different technical parameters like field strength and other. Our sample consists predominantly of invasive ductal carcinomas. Therefore, this study could not compare DCE MRI values between different tumor types. Presumably, other types like lobular or mucinous carcinomas may have different DCE MRI parameters than ductal carcinomas.

\section{Conclusion}

Our multicenter study showed that DCE MRI parameters $\mathrm{K}_{\text {trans }}, \mathrm{K}_{\mathrm{ep}}$, and $\mathrm{V}_{\mathrm{e}}$ cannot be used as a reliable surrogate marker for hormone receptor status, tumor stage and grade in BC. $\mathrm{K}_{\text {trans }}$ correlated moderately with expression of KI67 and may discriminate lesions with high and lower proliferation activity.

\section{Availability of Data and Materials}

The data that support the findings of this study are available from professor Surov but restrictions apply to the availability of these data, which were used under license for the current study, and so are not publicly available. Data are however available from the authors upon reasonable request and with permission of professor Surov.

\section{Conflicts of Interest}

The Authors declare no conflicts of interest.

\section{Authors' Contributions}

Concept design: Surov A.; Data collection: Kim J. Y., Aiello M., Huang W., Yankeelov T.E.; Statistical analysis: Wienke A.; Manuscript writing: Surov A., Pech M.; Final approval of manuscript: Kim J. Y., Aiello M., Huang W., Yankeelov T.E., Wienke A., Pech M.

\section{References}

1 DeSantis CE, Lin CC, Mariotto AB, Siegel RL, Stein KD, Kramer JL, Alteri R, Robbins AS and Jemal A: Cancer treatment and survivorship statistics, 2014. CA Cancer J Clin 64(4): 252271, 2014. PMID: 24890451. DOI: 10.3322/caac.21235

2 Youk JH, Son EJ, Chung J, Kim JA and Kim EK: Triplenegative invasive breast cancer on dynamic contrast-enhanced and diffusion-weighted MR imaging: comparison with other breast cancer subtypes. Eur Radiol 22(8): 1724-1734, 2012. PMID: 22527371. DOI: 10.1007/s00330-012-2425-2
3 Szabó BK, Aspelin P, Kristoffersen Wiberg M, Tot T and Boné $\mathrm{B}$ : Invasive breast cancer: correlation of dynamic MR features with prognostic factors. Eur Radiol 13(11): 2425-2435, 2003. PMID: 12898176. DOI: 10.1007/s00330-003-2000-y

4 Suo S, Cheng F, Cao M, Kang J, Wang M, Hua J, Hua X, Li L, $\mathrm{Lu} \mathrm{Q}$, Liu J and Xu J: Multiparametric diffusion-weighted imaging in breast lesions: Association with pathologic diagnosis and prognostic factors. J Magn Reson Imaging 46(3): 740-750, 2017. PMID: 28139036. DOI: 10.1002/jmri.25612

5 Jeh SK, Kim SH, Kim HS, Kang BJ, Jeong SH, Yim HW and Song BJ: Correlation of the apparent diffusion coefficient value and dynamic magnetic resonance imaging findings with prognostic factors in invasive ductal carcinoma. J Magn Reson Imaging 33(1): 102-109, 2011. PMID: 21182127. DOI: 10.1002/ jmri.22400

6 Mori N, Ota H, Mugikura S, Takasawa C, Ishida T, Watanabe G, Tada H, Watanabe M, Takase K and Takahashi S: Luminal-type breast cancer: correlation of apparent diffusion coefficients with the Ki-67 labeling index. Radiology 274(1): 66-73, 2015. PMID: 25203132. DOI: $10.1148 /$ radiol.14140283

7 Kato F, Kudo K, Yamashita H, Wang J, Hosoda M, Hatanaka $\mathrm{KC}$, Mimura R, Oyama-Manabe $\mathrm{N}$ and Shirato H: Differences in morphological features and minimum apparent diffusion coefficient values among breast cancer subtypes using 3-tesla MRI. Eur J Radiol 85(1): 96-102, 2016. PMID: 26724653. DOI: 10.1016/j.ejrad.2015.10.018

8 Sharma U, Sah RG, Agarwal K, Parshad R, Seenu V, Mathur SR, Hari S and Jagannathan NR: Potential of diffusion-weighted imaging in the characterization of malignant, benign, and healthy breast tissues and molecular subtypes of breast cancer. Front Oncol 6: 126, 2016. PMID: 27242965. DOI: 10.3389/fonc. 2016.00126

9 Surov A, Clauser P, Chang YW, Li L, Martincich L, Partridge SC, Kim JY, Meyer HJ and Wienke A: Can diffusion-weighted imaging predict tumor grade and expression of Ki-67 in breast cancer? A multicenter analysis. Breast Cancer Res 20(1): 58, 2018. PMID: 29921323. DOI: 10.1186/s13058-018-0991-1

10 Surov A, Chang YW, Li L, Martincich L, Partridge SC, Kim JY and Wienke A: Apparent diffusion coefficient cannot predict molecular subtype and lymph node metastases in invasive breast cancer: a multicenter analysis. BMC Cancer 19(1): 1043, 2019. PMID: 31690273. DOI: 10.1186/s12885-019-6298-5

11 Koo HR, Cho N, Song IC, Kim H, Chang JM, Yi A, Yun BL and Moon WK: Correlation of perfusion parameters on dynamic contrast-enhanced MRI with prognostic factors and subtypes of breast cancers. J Magn Reson Imaging 36(1): 145-151, 2012. PMID: 22392859. DOI: 10.1002/jmri.23635

12 Kang SR, Kim HW and Kim HS: Evaluating the relationship between dynamic contrast-enhanced MRI (DCE-MRI) parameters and pathological characteristics in breast cancer. $\mathbf{J}$ Magn Reson Imaging 52(5): 1360-1373, 2020. PMID: 32524658. DOI: $10.1002 /$ jmri.27241

13 Nagasaka K, Satake H, Ishigaki S, Kawai H and Naganawa S: Histogram analysis of quantitative pharmacokinetic parameters on DCE-MRI: correlations with prognostic factors and molecular subtypes in breast cancer. Breast Cancer 26(1): 113-124, 2019. PMID: 30069785. DOI: 10.1007/s12282-018-0899-8

14 Shin JK and Kim JY: Dynamic contrast-enhanced and diffusionweighted MRI of estrogen receptor-positive invasive breast cancers: Associations between quantitative MR parameters and 
Ki-67 proliferation status. J Magn Reson Imaging 45(1): 94-102, 2017. PMID: 27313102. DOI: 10.1002/jmri.25348

15 Afshari Mirak S, Mohammadian Bajgiran A, Sung K, Asvadi NH, Markovic D, Felker ER, Lu D, Sisk A, Reiter RE and Raman SS: Dynamic contrast-enhanced (DCE) MR imaging: the role of qualitative and quantitative parameters for evaluating prostate tumors stratified by Gleason score and PI-RADS v2 . Abdom Radiol (NY) 45(7): 2225-2234, 2020. PMID: 31549211. DOI: $10.1007 / \mathrm{s} 00261-019-02234-6$

16 Oto A, Yang C, Kayhan A, Tretiakova M, Antic T, SchmidTannwald C, Eggener S, Karczmar GS and Stadler WM: Diffusion-weighted and dynamic contrast-enhanced MRI of prostate cancer: correlation of quantitative MR parameters with Gleason score and tumor angiogenesis. AJR Am J Roentgenol 197(6): 1382-1390, 2011. PMID: 22109293. DOI: 10.2214/ AJR.11.6861

17 Jiang JS, Hua Y, Zhou XJ, Shen DD, Shi JL, Ge M, Geng QN and Jia ZZ: Quantitative assessment of tumor cell proliferation in brain gliomas with dynamic contrast-enhanced MRI. Acad Radiol 26(9): 1215-1221, 2019. PMID: 30416002. DOI: 10.1016/j.acra.2018.10.012

18 Meyer HJ, Wienke A and Surov A: Correlation between $\mathrm{K}_{\text {trans }}$ and microvessel density in different tumors: a meta-analysis. Anticancer Res 38(5): 2945-2950, 2018. PMID: 29715121. DOI: 10.21873/anticanres.12543

19 Surov A, Meyer HJ, Gawlitza M, Höhn AK, Boehm A, Kahn T and Stumpp P: Correlations between DCE MRI and histopathological parameters in head and neck squamous cell carcinoma. Transl Oncol 10(1): 17-21, 2017. PMID: 27888709. DOI: $10.1016 /$ j.tranon.2016.10.001
20 Liu F, Wang M and Li H: Role of perfusion parameters on DCEMRI and ADC values on DWMRI for invasive ductal carcinoma at 3.0 Tesla. World J Surg Oncol 16(1): 239, 2018. PMID: 30577820. DOI: 10.1186/s12957-018-1538-8

21 Petrelli F, Viale G, Cabiddu M and Barni S: Prognostic value of different cut-off levels of Ki-67 in breast cancer: a systematic review and meta-analysis of 64,196 patients. Breast Cancer Res Treat 153(3): 477-491, 2015. PMID: 26341751. DOI: 10.1007/ s10549-015-3559-0

22 Kim JY, Kim SH, Kim YJ, Kang BJ, An YY, Lee AW, Song BJ, Park YS and Lee HB: Enhancement parameters on dynamic contrast enhanced breast MRI: do they correlate with prognostic factors and subtypes of breast cancers? Magn Reson Imaging 33(1): 72-80, 2015. PMID: 25179138. DOI: 10.1016/j.mri. 2014.08.034

23 Carbonaro LA: Can we use MR-mammography to predict nodal status? Eur J Radiol 81 Suppl 1: S17-S18, 2012. PMID: 23083577. DOI: 10.1016/S0720-048X(12)70007-4
Received August 26, 2021

Revised October 3, 2021

Accepted October 12, 2021 